\title{
Discovery of X-ray flaring activity in the Arches cluster ${ }^{\star}$
}

\author{
R. Capelli ${ }^{1}$, R. S. Warwick ${ }^{2}$, N. Cappelluti ${ }^{1}$, S. Gillessen ${ }^{1}$, P. Predehl ${ }^{1}$, D. Porquet ${ }^{3}$, and S. Czesla ${ }^{4}$ \\ ${ }^{1}$ Max-Planck-Institut für Extraterrestrische Physik, Giessenbachstrasse 1, 85748 Garching, Germany \\ e-mail: capelli@mpe.mpg. de \\ 2 Department of Physics and Astronomy, University of Leicester, Leicester LE1 7RH, UK \\ 3 Observatoire Astronomique de Strasbourg, Université de Strasbourg, CNRS, UMR 7550, 11 rue de l'Université, \\ 67000 Strasbourg, France \\ ${ }^{4}$ Hamburger Sternwarte, Universität Hamburg, Gojenbergsweg 112, 21029 Hamburg, Germany \\ Received 15 September 2010 / Accepted 16 November 2010
}

\begin{abstract}
Context. We present a study of the Arches cluster based on XMM-Newton observations performed over the past 8 years. Unexpectedly, we find that the X-ray emission associated with the cluster experienced a marked brightening in March/April 2007.

Aims. We investigate the origin of both the X-ray continuum emission emanating from the star cluster and the flare.

Methods. To study the time variability of the total X-ray flux, we stacked the PN and MOS data of observations performed within a time interval of a few days leading to the detection of the flaring episode. We then constructed two spectral datasets, one corresponding to the flare interval (March/April 2007) and another to the normal quiescent state of the source.

Results. The X-ray light curve of the Arches cluster shows, with high significance $(8.6 \sigma)$, a $70 \%$ increase in the X-ray emission in the March/April 2007 timeframe followed by a decline over the following year to the pre-flare level; the short-term duration of the flare is constrained to be longer than four days. The temperature and the line-of-sight column density inferred from the flare spectrum do not differ from those measured in the normal activity state of the cluster, suggesting that the flux enhancement is thermal in origin. Conclusions. We attribute the X-ray variability to in situ stellar activity: early-type stars may be responsible for the flare via wind collisions, whereas late-type stars may contribute by means of magnetic reconnection. These two possibilities are discussed.
\end{abstract}

Key words. stars: flare - galaxy: center - X-rays: stars

\section{Introduction}

Three massive star clusters are located at the Galactic center (hereafter GC) region: the central (IRS 16) star cluster, the Arches cluster (hereafter AC), and the Quintuplet cluster (e.g., Morris \& Serabyn 1996). Whereas all three are very bright in the infrared band (e.g. Becklin \& Neugebauer 1975; Nagata et al. 1995), their X-ray properties differ markedly. Both IRS16 and the Quintuplet cluster are not particularly bright in X-rays with $L_{\mathrm{X}} \sim 10^{33} \mathrm{erg} / \mathrm{s}$ (Baganoff et al. 2003; Law \& Yusef-Zadeh 2004). In constrast, the AC has a relatively high X-ray luminosity $\left(L_{X} \sim 10^{34} \mathrm{erg} / \mathrm{s}\right)$ (e.g. Yusef-Zadeh et al. 2002). Although the total mass of each of these clusters is similar at about $10^{4} M_{\odot}$ (Figer 2004), the size of the clusters are different, with the AC being the most compact. The X-ray diffuse emission from the $\mathrm{AC}$ has been investigated in a number of published papers over the last decade (i.e. Yusef-Zadeh et al. 2002; Wang et al. 2006; Tsujimoto et al. 2007). All these studies confirm the co-existence in the cluster of both thermal and non-thermal radiation. The former is thought to be due largely to multiple interactions between the strong winds from massive stars, with the high stellar concentration in the AC providing an explanation of why its X-ray luminosity is enhanced (e.g. Cantó et al. 2000; Raga et al. 2001). Irrefutably, the AC, which is the densest cluster in the Galaxy with a core density of $10^{5} M_{\odot} / \mathrm{pc}^{3}$, provides an excellent opportunity to study stellar processes, within the environment of a massive star cluster, that lead to particle acceleration and X-ray emission. The study of these massive star clusters is of particu-

* Table 1 is only available in electronic form at http: //wWw . aanda.org lar interest in the context of the chemical enrichment of the GC region and their overall contribution to the high energy activity that characterises the innermost zone of the Galaxy.

\section{Observations and data reduction}

We selected archival XMM-Newton observations of the Galactic center region targeted at $\mathrm{Sgr} \mathrm{A}^{*}$. We reprocessed data from both the PN and MOS cameras (Strüder et al. 2001; Turner et al. 2001) with the tasks EPPROC and EMPROC in the Science Analysis Software version 9.0. The main properties of the XMM-Newton observations employed in the present work are reported in Table 1. We selected good time intervals (GTI) corresponding to periods of relatively low internal background. For this purpose, we compiled the 10-12 keV lightcurve of the full field of view and selected the time intervals for which the count rate was lower than a certain threshold. Since the selected observations were performed in different conditions of inter$\mathrm{nal} /$ particle background and orbital phase, we investigated all the lightcurves independently and selected an appropriate threshold count rate for each. The thresholds used in screening the data are reported in Table 1; in practice, these thresholds were selected so as to exclude all the peaks in the 10-12 keV full-field lightcurve. Throughout our analysis, we have only selected single and double events (PATTERN $\leq 4)$ for the PN and up to quadruple events for the MOS1 and MOS2 (PATTERN $\leq 12$ ) cameras. Similarly, only events tagged as real X-rays $(\mathrm{FLAG}==0)$ were utilised.

It was evident that the source was seen in an anomalous state in the three 2007 observations (separated by four days). For this reason, we considered the March/April 2007 observations (i.e., 


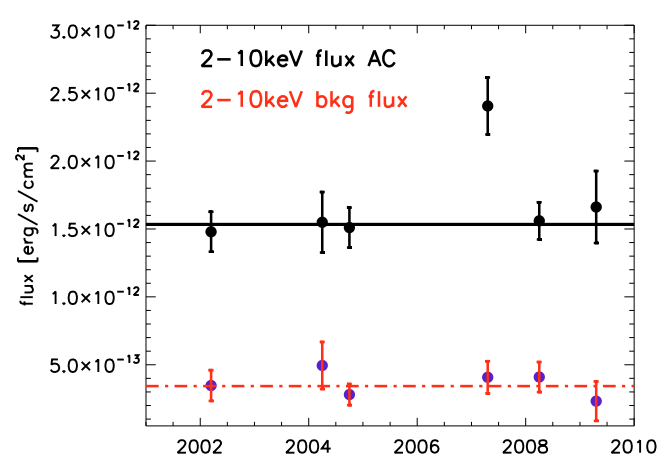

Fig. 1. The 2-10 keV X-ray lightcurve of the AC extending over an eight year period. The horizontal line is the weighted mean excluding the March/April 2007 flare. The red points show the lightcurve for the background, where the weighted mean is shown with a dashed red line.

the flare dataset) separately from the other observations. The region considered for the accumulation of the count rate spectrum of the source is an ellipse centered at RA $=17: 45: 50.358$ Dec $=-28: 49: 20.08$, with axes of $0.32^{\prime} \times 0.30^{\prime}$. We chose an elliptical region in order to enclose most of the X-ray diffuse emission in the AC (see contours in Fig. 2 in Yusef-Zadeh et al. 2002). Similarly a circular region of radius $0.4^{\prime}$, centered on $\mathrm{RA}=17: 45: 44.081 \mathrm{Dec}=-28: 49: 25.72$ was used to determine the background. Spectra corresponding to multiple observations were produced by stacking the data, using the tools MATHPHA, ADDRMF and ADDARF. The channels in each spectrum were grouped with the GRPPHA tool in order to have a minimum of 20 counts/bin, validating the use of the $\chi^{2}$ statistic.

\section{Results}

\subsection{The $X$-ray lightcurve}

To search for evidence of temporal variability in the X-ray emission from the AC, we constructed a "long-term" X-ray lightcurve based on six observation epochs, which in the majority of cases encompassed more than one individual observation. These were February 2002 (1 obs), March 2004 (2 obs), August/September 2004 (2 obs), March/April 2007 (3 obs), March 2008 (1 obs), and April 2009 (3 obs) - see Table 1.

The resulting $2-10 \mathrm{keV}$ lightcurve is shown in Fig. 1. Here the flux values are derived from model fits to the stacked spectra for each epoch using the average spectrum for the normal state (see below); more specifically, the points are the weighted mean of the PN and MOS values, corrected for absorption. A high state or flaring episode is evident in the 2007 measurements. As a check, we also considered the net source count rates measured in the individual cameras, which confirmed that this flaring is very likely a feature of the lightcurves. To quantify the statistical significance of this flare, we compared the weighted mean of the other five measurements in Fig. 1 of $1.53 \pm 0.07 \times$ $10^{-12} \mathrm{erg} / \mathrm{cm}^{2} / \mathrm{s}$ with the flux measured in March/April 2007 of $2.4 \pm 0.2 \times 10^{-12} \mathrm{erg} / \mathrm{cm}^{2} / \mathrm{s}$; the variability is significant at the level of $8.6 \sigma$.

To check whether the observed flare was produced by systematics in the background, we also built the lightcurve of the flux measured from the region used for the background (the red points in Fig. 1). For these data, the $\chi_{\text {red }}^{2}$ with respect to the weighted mean of the measurements $\left(3.4 \pm 0.4 \times 10^{-13} \mathrm{erg} / \mathrm{cm}^{2} / \mathrm{s}\right)$ is 0.7 , fully consistent with a constant background.

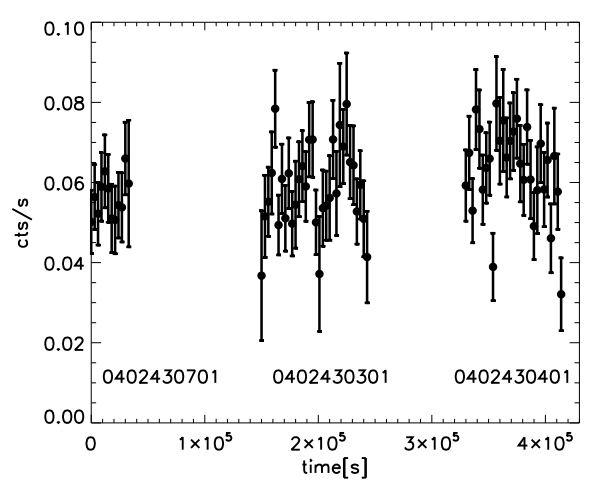

Fig. 2. The lightcurve from the March-April 2007 observations. The energy range is $2-10 \mathrm{keV}$ and the time binning is set to $3 \mathrm{ks}$.

We have also studied the $2-10 \mathrm{keV}$ lightcurve of the individual March-April 2007 observations to look for evidence of short-term variability during the episode of enhanced activity. The result of this investigation is shown in Fig. 2. There is no clear evidence of a trend in this full lightcurve; a constant fit to the data gives a mean count rate of $0.059 \pm 0.001 \mathrm{cts} / \mathrm{s}$, with a $\chi_{\text {red }}^{2}=1.1$. Although there are some low-level short-term features apparent in the individual observations, we conclude that these are most likely due to background-subtraction errors, since similar effects are apparent in the lightcurves of other sources in the field of view. We conclude that the duration of the high state activity is longer than $\sim 4$ days.

\subsection{X-ray spectral analysis}

We first performed an analysis of the stacked PN and MOS X-ray spectra pertaining to the normal state of the AC. The spectral model comprised a collisionally ionized plasma (APEC, Smith et al. 2001) plus a non-thermal hard tail modeled by a power-law continuum with a photon index $\Gamma$. In the present investigation, we found it advantageous to fix $\Gamma$ to a value of 1 , thereby avoiding potential biases in the spectral modeling of multiple datasets with a different balance between the thermal and non-thermal contributions. This approach is consistent with earlier studies that generally found $\Gamma$ to be in the range $1-1.5$ (e.g., Wang et al. 2006; Tsujimoto et al. 2007) and consistent with the power-law slope characterising bright $6.4 \mathrm{keV}$ fluorescent line-emitting regions in the vicinity of the $\mathrm{AC}$ (which will be the subject of a later study). In the analysis, we also fixed the metallicity of the thermal plasma at $2 Z_{\odot}$ (e.g., Wang et al. 2006), since the spectral fitting of both the stacked and the single datasets did not usefully constrain this parameter. In this context, we note that there is strong evidence that the diffuse plasma in the GC region has a super-solar metallicity (e.g., Tanaka 2002; Koyama et al. 2007). Two gaussian lines were included to account for the neutral (or near-neutral) $\mathrm{Fe} \mathrm{K}_{\alpha}$ and $\mathrm{Fe} K_{\beta}$ lines, at 6.4 and $7.05 \mathrm{keV}$ respectively, the $K_{\beta} / \mathrm{K}_{\alpha}$ flux ratio being tied to 0.11 . Finally, all the components were subject to interstellar absorption (WABS model in XSPEC, Morrison \& McCammon 1983).

The results of the joint fitting of the PN and MOS spectra for the normal state of the source are reported in the second column of Table 2 and the corresponding spectra are shown in Fig. 3 (left panel). The best-fit temperature of the cluster was found to be $k T=1.7 \pm 0.1 \mathrm{keV}$, in good agreement with that previously measured with Suzaku (Tsujimoto et al. 2007) and Chandra (Wang et al. 2006). Moreover, the resulting total $2-10 \mathrm{keV}$ flux is $5.3 \pm 0.5 \times 10^{-13} \mathrm{erg} / \mathrm{cm}^{2} / \mathrm{s}\left(1.5 \pm 0.1 \times 10^{-12} \mathrm{erg} / \mathrm{cm}^{2} / \mathrm{s}\right.$ absorption 
Table 2. Results of the spectral fitting for the normal state and the net flare spectrum of the AC.

\begin{tabular}{ccc}
\hline \hline & NORMAL & FLARE \\
\hline$N_{\mathrm{H}}\left(10^{22} \mathrm{~cm}^{-2}\right)$ & $10.4 \pm 0.9$ & $10.2 \pm 2.1$ \\
$k T_{\mathrm{APEC}}(\mathrm{keV})$ & $1.7 \pm 0.1$ & $1.8 \pm 0.3$ \\
flux $_{\mathrm{APEC}}\left(10^{-5}\right)$ & $5.4 \pm 0.4$ & $5.2 \pm 0.9$ \\
$\Gamma_{\mathrm{POW}}$ & 1.0 & 1.0 \\
flux $_{\mathrm{POW}}\left(10^{-5}\right)$ & $1.3 \pm 0.1$ & - \\
$f_{\mathrm{Fe}_{1} K_{\alpha}}\left(10^{-6}\right)$ & $1.4 \pm 0.7$ & - \\
flux $\left(10^{-5}\right)$ & $7.0 \pm 0.5$ & $5.2 \pm 0.9$ \\
$\chi^{2} /$ d.o.f. & $234 / 1144$ & $246.5 / 852$ \\
\hline
\end{tabular}

Notes. The values are the weighted mean of the PN and MOS measurements. The fluxes $(2-10 \mathrm{keV})$ are in photons $/ \mathrm{cm}^{2} / \mathrm{s}$.

corrected), which translates into a luminosity of $3.8 \pm 0.4 \times$ $10^{33} \mathrm{erg} / \mathrm{s}$. To quantify the respective contributions of the thermal and non-thermal emission to the spectrum, we measured the fluxes associated with the different continuum components in the best fit model. For the $2-10 \mathrm{keV}$ band, the division was into an $85 \%$ contribution by the thermal emission and a $15 \%$ one by the hard non-thermal tail. Both the thermal and non-thermal components are very likely to be manifestations of in situ activity within the AC. More specifically, shocks produced by strong colliding stellar winds can both heat the plasma and supply high energy particles. Law \& Yusef-Zadeh (2004) proposed that energetic electrons within the cluster might be able to upscatter IR radiation from stars via the inverse Compton effect; in this case, the power-law component should contribute about a sixth of the total flux, in excellent agreement with the measurements.

Given the discovery of significant variability in the X-ray flux from the $\mathrm{AC}$, the next step was to investigate the spectrum of the enhancement. For this, we used the stacked 2007 dataset as the source event file, with the background taken to be the source spectrum of the AC during its normal state. The PN and MOS spectra corresponding to the flare (net flare spectra) are presented in Fig. 3 (right panel), in comparison to the normal state spectrum (left panel). The main difference between the spectra of the two states of the $\mathrm{AC}$ is in the $6.4 \mathrm{keV}$ fluorescent line from neutral (or near-neutral) Fe; the flare spectrum does not show significant emission at this energy. This clearly indicates that the $6.4 \mathrm{keV} \mathrm{Fe} \mathrm{K}_{\alpha}$ line has a different origin to the higher ionization $6.7 \mathrm{keV}$ iron line. This observational evidence points to a thermal origin for the flare. This was confirmed when we attempted to fit the flare spectra with the same model as applied to the normal state data. For the flare, we found that the nonthermal power-law and the low-ionization state lines of Fe (at 6.4 and $7.05 \mathrm{keV}$ ) were then no longer required. The flare spectrum is described well by a thermal plasma with $k T=1.8 \pm 0.3 \mathrm{keV}$ and a total flux of $3.7 \pm 0.6 \times 10^{-13} \mathrm{erg} / \mathrm{cm}^{2} / \mathrm{s}$; in terms of $2-10 \mathrm{keV}$ $\mathrm{X}$-ray flux, the flare represents an increase over the normal state activity of about $70 \pm 13 \%$.

We highlight that the temperature and the $N_{\mathrm{H}}$ values inferred for the flare spectra are in excellent agreement with the values derived from the normal state spectrum (see Fig. 3 and Table 2). It would seem that the variability in the cluster is produced by an increase in the intensity of the thermal emission, while the other physical observables, namely the temperature and $N_{\mathrm{H}}$, remain unchanged. The emission measure (EM) of this X-ray activity (the flare component) was estimated from the normalization of the APEC component to be some $1.2 \times 10^{57} \mathrm{~cm}^{-3}$.

\section{Discussion}

We have investigated the X-ray properties of the AC and reported the first detection of significant variability in its hot thermal emission. The bulk of this component is thought to arise from the thermalization of the strong winds expelled by massive stars. Cantó et al. (2000) calculated the expected temperature of such a cluster wind to be

$k T_{\text {cluster }}=1.3\left(\frac{v_{\mathrm{w}}}{10^{3} \mathrm{kms}^{-1}}\right)^{2} \mathrm{keV}$,

where $v_{\mathrm{w}}$ is the terminal velocity for the stellar wind, which is assumed to be the same for all the stars within the cluster. The temperature we measured in the $\mathrm{AC}$ is $1.7 \pm 0.4 \mathrm{keV}$, which requires a terminal velocity of $1100-1200 \mathrm{~km} \mathrm{~s}^{-1}$, in good agreement with the actual wind velocities reported by Cotera et al. (1996). In the normal state of the AC, the EM of this plasma is $1.4 \pm 0.3 \times 10^{57} \mathrm{~cm}^{-3}$. If we model the $\mathrm{AC}$ as a sphere of $0.7 \mathrm{pc}$ radius and set $n_{\mathrm{e}} \sim n_{\mathrm{H}}$, the inferred electron density is 1$10 \mathrm{~cm}^{-3}$. This value again compares very well to theoretical estimates obtained by modeling the X-ray emission of dense clusters of massive stars (see middle panel of Fig. 3 in Cantó et al. 2000). Using the superior spatial resolution of the Chandra telescope, Yusef-Zadeh et al. (2002) identified three distinct spatial components in the $\mathrm{AC}$; components $\mathrm{A} 1$ and $\mathrm{A} 2$ were found to have strong thermal emission, whereas A3 component was found to be extended and more elongated towards the south-west of the cluster core. Overall the diffuse emission is characterized by a highly significant $\mathrm{Fe} \mathrm{K}_{\alpha}$ line at $6.4 \mathrm{keV}$ corresponding to neutral or low ionization gas. Because of the lower angular resolution of $X M M-N e w t o n$, we could not resolve these three components and study them separately but simply analyzed a composite spectrum. In a similar way, Tsujimoto et al. (2007) used Suzaku data to study a 1.4 arcmin wide region centred on the cluster and derive spectral parameters very similar to those reported here.

The non-thermal radiation associated with the $\mathrm{AC}$ is most likely attributed to the presence of a sea of cosmic rays permeating the whole region within the cluster and the surroundings; Valinia et al. (2000) showed that the diffuse emission from the Galactic ridge can be explained in terms of low energy cosmic rays (LECRs) producing both the continuum and the low ionization lines from the different elements. However, the slope of the non-thermal continuum emission found by these authors is about 1.3-1.4, somewhat steeper than the value we adopt. This difference may reflect the extreme conditions in the vicinity of the AC itself. The collisions between powerful stellar winds with terminal velocities at or in excess of $1200 \mathrm{~km} \mathrm{~s}^{-1}$ can create shocks that accelerate $\mathrm{CRs}$ up to $\mathrm{keV}$ energies, thereby leading to a flattening of the spectrum in the high energy domain via either bremsstrahlung or inverse Compton emission.

Here we report an unexpectedly high state of activity in the $\mathrm{AC}$, although the XMM-Newton off-axis PSF does not allow us to directly locate the flare emission within the spatial substructure observed in the AC with Chandra (Yusef-Zadeh et al. 2002). The spectrum of the AC in this flaring state is well modeled as a hot plasma with $k T=1.8 \pm 0.3 \mathrm{keV}$. This, and the presence in the spectrum of the high ionization lines of elements such as $\mathrm{S}, \mathrm{Ar}, \mathrm{Ca}$, and Fe implies a stellar origin for the observed flux increase. The longest detected stellar coronal flares to date had a duration of nine days. For the AC, we can set a lower limit to the duration of the enhanced luminosity of $\sim 4$ days. This is consistent with a coronal origin for the flare. On the other hand, we note that if the flare duration were a few months, its origin would be very unlikely to be coronal. Assuming a distance to 

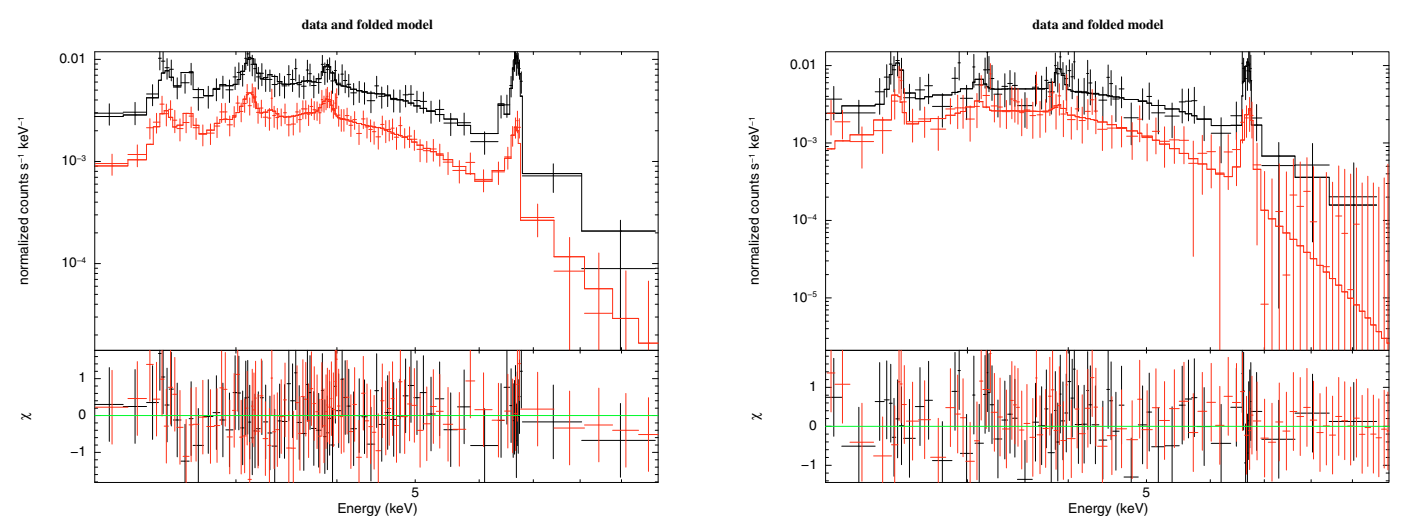

Fig. 3. The 2-10 keV X-ray spectra of the AC as measured in the PN (black) and MOS (red) cameras. Left panel: the spectrum measured in the normal state. Right panel: the net flare spectrum measured during the flaring episode. In both cases, the best fitting spectral model and the corresponding residuals are also shown.

the GC of $8 \mathrm{kpc}$ (Gillessen et al. 2009), the estimated EM for the AC flare is $1.2 \times 10^{57} \mathrm{~cm}^{-3}$, very significantly higher than the values typically measured in stellar X-ray flares $\left(\sim 10^{55} \mathrm{~cm}^{-3}\right)$ associated with late-type stars (e.g., Güdel 2004; Güdel \& Nazé 2009). The X-ray flares from late-type stars observed to date exhibit a strong correlation between the peak temperature and the EM. Aschwanden et al. (2008) found that the EM in a stellar flare is proportional to $T^{4.5}$; according to this relation, an EM of $1.2 \times 10^{57} \mathrm{~cm}^{-3}$ yields a temperature well above $10^{8} \mathrm{~K}(8.6 \mathrm{keV})$, and well beyond the value measured in the AC flare (1.8 keV corresponding to $\sim 2 \times 10^{7} \mathrm{~K}$ ). We therefore dismiss the late-type star hypothesis for the origin of the flare.

An alternative scenario that could account for the detected variability entails early-type stars, possibly in binary configurations. Either an orbital modulation of the system or a sudden eruption by one star might produce the observed high state activity. In both cases, the density and the velocities of the colliding stellar winds might change, thus producing an enhancement in the X-ray flux. The X-ray emission arising from equally strong colliding winds is inversely proportional to the binary separation $\left(D^{-1}\right)$; for stellar parameters such as those found in the AC $\left(\dot{M} \sim 10^{-5} M_{\odot} / \mathrm{yr}\right.$ and $\left.v_{\infty} \sim 1200 \mathrm{~km} \mathrm{~s}^{-1}\right)$ the X-ray luminosity created by colliding winds is about $10^{32-33} \mathrm{erg} / \mathrm{s}$, for $D \gtrsim 10-$ $500 \mathrm{AU}$. In this context, it is plausible that the enhanced X-ray activity might mark the periastron passage of a system with an eccentric orbit. Mauerhan et al. (2010) reported X-ray emission from massive stars in the GC region. Among the sources studied in their sample, four are located in the AC, two of them being inside the XMM PSF and good candidates for the location of the flaring event. Another example of variability involving early-type stars can be found in the binary system $\eta$ Carinae; this has been measured to be variable with an increase in the $\mathrm{X}$-ray luminosity of about $80 \%$. In this supermassive colliding wind binary, the X-ray luminosity reaches $10^{34} \mathrm{erg} / \mathrm{s}$ and the flare timescales range between a few days and about 100 days (Moffat \& Corcoran 2009). A likely explanation of this variability is the interaction of clumpy winds; this process has been observed to produce an X-ray luminosity 1.8 times brighter than the normal level, with a spread in the duration of the high state activity between $\sim 3$ and 50 days, characteristics that might equally well describe the AC flare.

Here we propose that the colliding wind binary hypothesis provides the most plausible explanation of the AC flare. On the basis of the lightcurve, the upper limit to the duration of the measured flare is about four years. Assuming a wind velocity of $1200 \mathrm{~km} \mathrm{~s}^{-1}$, the linear size of the region intersected by a propagating density enhancement over a four year period is about $0.005 \mathrm{pc}$, whereas the mean distance between stars in a dense environment such as the AC core can be estimated to be $\sim 0.1 \mathrm{pc}$. This argument lends further support to the stellar origin of this $\mathrm{X}$-ray activity. Presumably, these interactions are quite probable in a dense cluster such as the AC, especially in the southern component, where the highest concentration of massive colliding wind binaries has been reported (Yusef-Zadeh et al. 2002).

In the future it will be of great importance to characterise more precisely the temporal variability exhibited by massive star clusters such as the AC, both from the perspective of the underlying processes and in the context of the contribution of these events to the high-energy budget of the whole GC region.

\section{References}

Aschwanden, M. J., Stern, R. A., \& Güdel, M. 2008, ApJ, 672, 659 Baganoff, F. K., Maeda, Y., Morris, M., et al. 2003, ApJ, 591, 891 Becklin, E. E., \& Neugebauer, G. 1975, ApJ, 200, L71

Cantó, J., Raga, A. C., \& Rodríguez, L. F. 2000, ApJ, 536, 896

Cotera, A. S., Erickson, E. F., Colgan, S. W. J., et al. 1996, ApJ, 461, 750

Figer, D. F. 2004, in The Formation and Evolution of Massive Young Star Clusters, ed. H. J. G. L. M. Lamers, L. J. Smith, \& A. Nota, ASP Conf. Ser., 322,49

Gillessen, S., Eisenhauer, F., Trippe, S., et al. 2009, ApJ, 692, 1075

Güdel, M. 2004, A\&ARv, 12, 71

Güdel, M., \& Nazé, Y. 2009, A\&ARv, 17, 309

Koyama, K., Hyodo, Y., Inui, T., et al. 2007, PASJ, 59, 245

Law, C., \& Yusef-Zadeh, F. 2004, ApJ, 611, 858

Mauerhan, J. C., Muno, M. P., Morris, M. R., Stolovy, S. R., \& Cotera, A. 2010, ApJ, 710, 706

Moffat, A. F. J., \& Corcoran, M. F. 2009, ApJ, 707, 693

Morris, M., \& Serabyn, E. 1996, ARA\&A, 34, 645

Morrison, R., \& McCammon, D. 1983, ApJ, 270, 119

Nagata, T., Woodward, C. E., Shure, M., \& Kobayashi, N. 1995, AJ, 109, 1676

Raga, A. C., Velázquez, P. F., Cantó, J., Masciadri, E., \& Rodríguez, L. F. 2001, ApJ, 559, L33

Smith, R. K., Brickhouse, N. S., Liedahl, D. A., \& Raymond, J. C. 2001, ApJ, 556, L91

Strüder, L., Briel, U., Dennerl, K., et al. 2001, A\&A, 365, L18

Tanaka, Y. 2002, A\&A, 382, 1052

Tsujimoto, M., Hyodo, Y., \& Koyama, K. 2007, PASJ, 59, 229

Turner, M. J. L., Abbey, A., Arnaud, M., et al. 2001, A\&A, 365, L27

Valinia, A., Tatischeff, V., Arnaud, K., Ebisawa, K., \& Ramaty, R. 2000, ApJ, 543,733

Wang, Q. D., Dong, H., \& Lang, C. 2006, MNRAS, 371, 38

Yusef-Zadeh, F., Law, C., Wardle, M., et al. 2002, ApJ, 570, 665

\section{Page 5 is available in the electronic edition of the journal at http: //wWw . aanda.org}


R. Capelli et al.: X-ray flaring activity in the Arches cluster

Table 1. The selected datasets identified by means of both the OBSID and the observation date.

\begin{tabular}{ccccc}
\hline \hline OBSID & $\begin{array}{c}\text { Obs Date } \\
\text { yyyy-mm-dd }\end{array}$ & $\begin{array}{c}\text { PN } \\
\text { cut/GTI/exp }\end{array}$ & $\begin{array}{c}\text { MOS1 } \\
\text { cut/GTI/exp }\end{array}$ & $\begin{array}{c}\text { MOS2 } \\
\text { cut/GTI/exp }\end{array}$ \\
\hline 0111350101 & $2002-02-26$ & $0.8 / 38.590 / 40.030$ & $0.5 / 42.262 / 52.105$ & $0.5 / 41.700 / 52.120$ \\
0202670501 & $2004-03-28$ & $2.0 / 13.320 / 101.170$ & $1.0 / 33.070 / 107.784$ & $1.0 / 30.049 / 108.572$ \\
0202670601 & $2004-03-30$ & $2.0 / 25.680 / 112.204$ & $1.0 / 32.841 / 120.863$ & $1.0 / 35.390 / 122.521$ \\
0202670701 & $2004-08-31$ & $1.0 / 59.400 / 127.470$ & $0.5 / 80.640 / 132.469$ & $0.5 / 84.180 / 132.502$ \\
0202670801 & $2004-09-02$ & $1.0 / 69.360 / 130.951$ & $0.5 / 94.774 / 132.997$ & $0.5 / 98.757 / 133.036$ \\
0402430301 & $2007-04-01$ & $1.5 / 61.465 / 101.319$ & $0.8 / 61.002 / 93.947$ & $0.8 / 62.987 / 94.022$ \\
0402430401 & $2007-04-03$ & $1.5 / 48.862 / 93.594$ & $0.8 / 40.372 / 97.566$ & $0.8 / 41.317 / 96.461$ \\
0402430701 & $2007-03-30$ & $1.5 / 32.337 / 32.338$ & $0.8 / 26.720 / 33.912$ & $0.8 / 27.685 / 33.917$ \\
0505670101 & $2008-03-23$ & $1.25 / 74.216 / 96.601$ & $0.5 / 73.662 / 97.787$ & $0.5 / 74.027 / 97.787$ \\
0554750401 & $2009-04-01$ & $1.0 / 30.114 / 38.034$ & $0.5 / 32.567 / 39.614$ & $0.5 / 33.802 / 39.619$ \\
0554750501 & $2009-04-03$ & $1.0 / 36.374 / 42.434$ & $0.5 / 41.376 / 44.016$ & $0.5 / 41.318 / 44.018$ \\
0554750601 & $2009-04-05$ & $1.0 / 28.697 / 32.837$ & $0.5 / 37.076 / 38.816$ & $0.5 / 36.840 / 38.818$ \\
\hline
\end{tabular}

Notes. For each instrument, we report the threshold in the 10-12 keV lightcurve (counts/s) used for GTI selection, the total GTI exposure (ks), and the nominal observation duration $(\mathrm{ks})$. 